\title{
Polymorphisme génétique du métabolisme hépatique des médicaments
}

L'existence d'un polymorphisme génétique explique les importantes différences observées entre les individus dans leur métabolisme des médicaments. L'identification des mécanismes en cause devrait faciliter la prévention des hépatites médicamenteuses.

\section{Dominique Larrey Chef de Clinique}

\section{ADRESSE}

D. Larrey : Unité de recherches de physiopathologie hépatique, Inserm U 24, hôpital Beaujon, 'organisme est exposé en permanence à de nombreux xénobiotiques naturels auxquels s'ajoute un nombre croissant de médicaments. Certains xénobiotiques sont très peu solubles dans l'eau et ne peuvent être excrétés tels quels dans la bile ou dans les urines. Pour tourner cette difficulté, l'organisme dispose de systèmes enzymatiques permettant de transformer ces xénobiotiques liposolubles en métabolites hydrosolubles, facilement éliminés dans les urines ou les fécès. Ces réactions enzymatiques sont effectuées en grande partie dans le foie. Elles comportent des biotransformations de type I, comprenant des réactions d'oxydation, de réduction et d'hydrolyse, et des biotransformations de type II, comprenant diverses réactions de conjugaison [I]. La vitesse de ces réactions métaboliques est très variable d'un individu à l'autre. Cette variabilité du métabolisme est due, en partie, à divers facteurs physiologiques (âge, sexe), pathologiques (maladies du foie, de la thyroide, etc.), ou liés à l'environnement (régime alimen- taire, tabagisme, consommation d'alcool ou de médicaments) [I-3]. L'influence du contrôle génétique de la biotransformation des médicaments a été reconnue dès 1959 donnant ainsi naissance à une nouvelle discipline, la pharmacogénétique. Outre les déterminismes polygéniques donnant lieu à une distribution modale (monophasique) entre les divers individus, on a peu à peu découvert un certain nombre de déterminismes monogéniques qui aboutissent à une distribution bimodale (ou trimodale) des individus en métaboliseurs rapides, lents (ou intermédiaires). Le polymorphisme génétique est défini par l'existence d'une variation génétique responsable de l'existence de différents phénotypes, dont le moins fréquent, en général le phénotype déficitaire, n'est toutefois pas exceptionnel (de l'ordre de I à Io \% de la population, pour la plupart des médicaments). Cette définition exclut donc une mutation n'affectant que quelques familles isolées. Des exemples déjà anciens de polymorphismes génétiques sont l'hydrolyse de la succinylcholine par 
la cholinestérase, ou la N-acétylation de certains médicaments comme l'isoniazide ou l'hydralazine. Mais c'est surtout depuis 1977 que la pharmacogénétique a connu un grand développement avec la découverte d'un polymorphisme génétique touchant l'oxydation de certains médicaments par le cytochrome P-450 [4-7].

\section{La N-acétylation}

La $\mathrm{N}$-acétylation de l'isoniazide est l'exemple le plus connu des polymorphismes génétiques de l'acétylation des médicaments. La capacité d'acétylation est en effet le facteur essentiel qui détermine la concentration plasmatique d'isoniazide, pour une dose donnée. Son évaluation quantitative dans une large population a mis en évidence une distribution bimodale en acétyleurs rapides, et en acétyleurs lents [8]. L'étude de familles a montré que l'acétylation de l'isoniazide est contrôlée par une paire d'allèles autosomiques à un seul locus. Schématiquement, le caractère acétyleur rapide est dominant $(R)$ et le caractère acétyleur lent est récessif ( $r$ ) [8] : les sujets homozygotes (RR) et hétérozygotes $(R r)$ pour le trait acétyleur rapide ont le phénotype acétyleur rapide, alors que seuls les sujets homozygotes (rr) ont le phénotype acétyleur lent. De façon un peu plus fine, il semble qu'il soit en réalité possible de distinguer, dans le groupe des acétyleurs rapides, des sujets dont l'acétylation est particulièrement rapide, correspondant aux homozygotes (RR), et des sujets dont l'acétylation est un peu moins rapide, correspondant aux hétérozygotes ( $\mathrm{Rr}$ ) [9]. La prévalence du phénotype acétyleur rapide est très variable d'une population à l'autre : elle est de $18 \%$ seulement chez les Égyptiens, de 30 à $50 \%$ chez les Européens et les Blancs d'Amérique du Nord, de 50 à $65 \%$ chez les Noirs africains et américains, et dépasse $70 \%$ chez les Asiatiques, les Indiens d'Amérique du Sud et les Esquimaux [9, 10].

Le polymorphisme de la $\mathrm{N}$-acétylation touche non seulement l'isoniazide mais aussi de nombreux autres médicaments énumérés dans

$m / s n^{\circ} 7$, vol. 2, septembre 86 le tableau I. Les deux composés les plus utilisés actuellement pour déterminer la capacité d'acétylation sont l'isoniazide et la sulfadimidine. Deux autres méthodes récentes sont la détermination des métabolites urinaires de la dapsone ou de la caféine [9].

\section{Mécanisme biochimique}

La N-acétylation est effectuée par la $\mathrm{N}$-acétyltransférase, un enzyme cytosolique qui est principalement présent dans le foie et, à un moindre degré, dans la muqueuse de l'intestin grêle. La plus faible activité in vitro de la $\mathrm{N}$-acétyltransférase chez les acétyleurs lents pourrait être due à une diminution de la quantité d'enzyme ou bien à une modification de son affinité [9].

\section{Conséquences cliniques}

Une première conséquence est que les acétyleurs lents recevant une dose standard de médicament vont être exposés à des concentrations élevées de médicament non transformé. Il peut en résulter divers eff ets toxiques. C'est ainsi que les acétyleurs lents sont particulièrement exposés au risque de neuropathie périphérique induite par l'isoniazide, à l'apparition de lupus érythémateux disséminé induit par l'isoniazide, l'hydralazine, ou le procaïnamide, à divers phénomènes d'intolérance à la salicylazosulfapyridine (cyanose et hémolyse) ou à la phénelzine (somnolence et nausées) $[7,9]$. Une relation entre le phénotype acétyleur lent et le risque de cancer de la vessie induit par diverses arylamines a été suggérée $[7,9]$. Cependant, si cette relation apparaît significative chez les ouvriers professionnellement exposés à la benzidine $[7,9]$, elle n'a pas été clairement démontrée dans une population urbaine non sélectionnée, et uniquement exposée aux arylamines présentes dans l'environnement ou dans la fumée de tabac $[7,9]$. Même en l'absence de toute prise médicamenteuse, les sujets acétyleurs lents seraient plus fréquemment atteints de lupus érythémateux disséminés "spontanés" $[7,9]$. Les sujets atteints d'un déficit en glucose 6-phosphate déshydrogénase sont exposés à un risque accru d'hémolyse quand ils sont en outre acétyleurs lents [9]. Enfin, une interaction médicamenteuse entre l'isoniazide et la phénytoïne, se traduisant par des signes d'intolérance à la phénytoïne, est plus fréquemment observée chez les acétyleurs lents [9].

Une deuxième conséquence du phénotype d'acétylation est qu'à l'inverse, une dose standard du médicament peut être inefficace chez l'acétyleur rapide. En eff et, cette dose de médicament est calculée d'après la population globale qui est constituée d'acétyleurs lents ou rapides

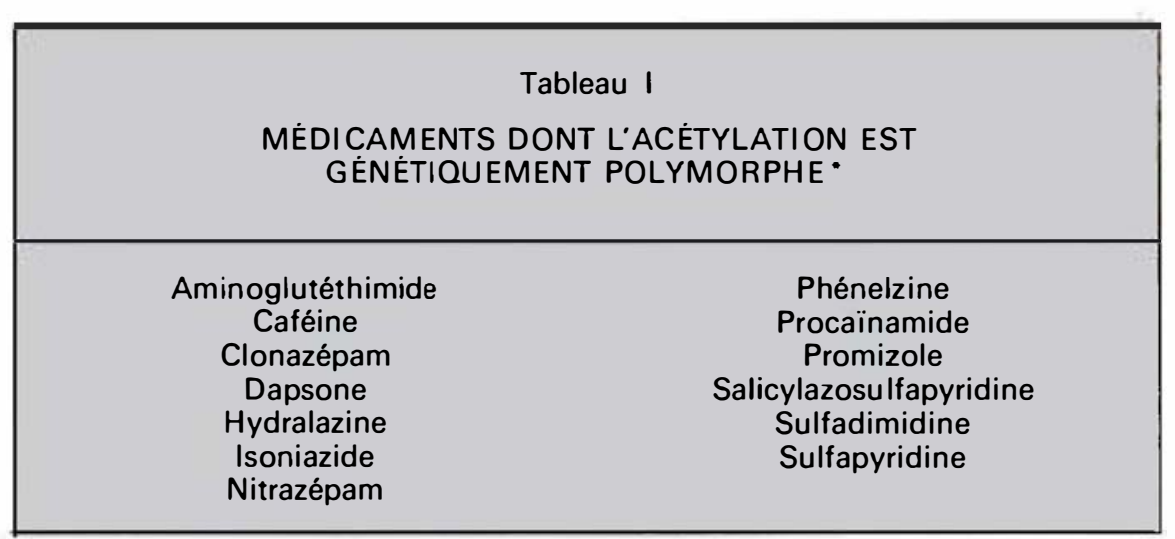

- D'après Weber et Hein [8]. 


\section{RÉFÉRENCES}

I. Park BK. Assessment of the drug metabolism capacity of the liver. Br 7 Clin Pharmacol 1982; 14: 631-51.

2. Larrey D, Branch RA. Clearance by the liver: current concepts in understanding the hepatic drug disposition of drugs. Semin Liver Dis 1983; 3: 285-97.

3. Vesell ES. The antipyrine test in clinical pharmacology: conceptions and misconceptions. Clin Pharmacol Ther 1979; 26: 275-86.

4. Maghoub A, Idle JR, Dring LG, Lancaster R, Smith RL. Polymorphic hydroxylation of debrisoquine in man. Lancet 1977; ii: 584-6.

5. Eichelbaum M. Defective oxidation of drugs: pharmacokinetics and therapeutic implications. Clin Pharmacokinet 1982; 7: 1-22.

6. Küpfer A, Preisig R. Inherited def ects of hepatic drug metabolism. Semin Liver Dis 1983; 3: 341-54.

7. Larrey D, Pessayre D, Benhamou JP. Polymorphisme génétique du métabolisme hépatique des médicaments. Gastroenterol Clin Biol 1985; 9: 522-31.

8. Evans DAP, Manley KA, McKusick VA Genetic control of isoniazid metabolism in man. Br Med 7 1960; 2: 485-91.

9. Weber WW, Hein DW. N-acetylation pharmacogenetics. Pharmacol Rev 1985; 37: 25-79.

I0. Kalow W. Ethnic differences in drug metabolism. Clin Pharmacokinet 1982; 7: 373-400.

I1. Mitchell JR, Thorgeirsson UP, Black M, et al. Increased incidence of isoniazid hepatitis in rapid acetylators: possible relation to hydrazin metabolites. Clin Pharmacol Ther 1975; 18: 70-9.

12. Billing BH. Bilirubin metabolism. In: Schiff L, SchiffE, eds. Diseases of the liver. Philadelphia: Lippincott, 1982: 349-78.

13. Billing BH. Twenty-five years of progress in bilirubin metabolism (1952-1977). Gut 1978; 19: 48I-9I.

14. Arias IM, Gartner LM, Cohen M, Ben Ezzer J, Levi AJ. Chronic non-hemolytic unconjugated hyperbilirubinemia with glucuronosyl transferase deficiency. Am 7 Med 1969; 47: 395-409.

15. Macklon AF, Savage RL, Rawlins MD. Gilbert's syndrome and drug metabolism. Clin Pharmacokinet 1979; 4: 223-32.

16. Kalow W, Tang BK, Kadar D, Endrenyi L, Chan FY. A method for studying drug metabolism in populations: racial differences in amobarbital metabolism. Clin Pharmacol Ther 1979; 26: 766-76.

17. Wang PP, Beaune P, Kaminsky LS, et al. Purification and characterization of six cytochrome P-450 isozymes from human liver

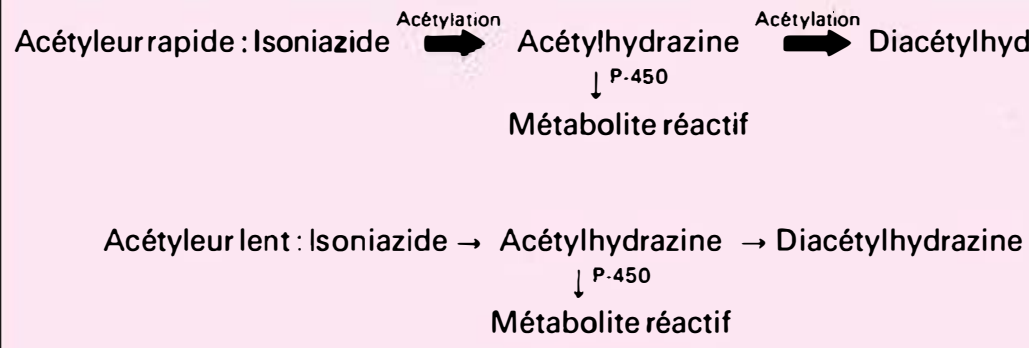

Figure 1. Métabolisme de l'isoniazide. L'isoniazide est d'abord acétylé en acétylisoniazide qui est rapidement hydrolysé en acide isonicotinique et acétylhydrazine. L'acétylhydrazine peut être acétylée à son tour en diacétylhydrazine ou être oxydée par le cytochrome $P$-450 en un métabolite réactif hépatotoxique. De ce fait, la concentration plasmatique maximale d'acétylhydrazine est finalement à peu près égale chez les acétyleurs lents et les acétyleurs rapides.

dans une proportion à peu près égale. Par exemple, les acétyleurs rapides ont besoin d'une dose plus élevée d'hydralazine, de dapsone et de phénelzine pour bénéficier d'un même effet thérapeutique $[7,9]$. En ce qui concerne l'hépatotoxicité de l'isoniazide, il a initialement été proposé que l'incidence des hépatites pourrait être plus forte chez les sujets acétyleurs rapides [II]. En réalité, il n'en est pas ainsi $[7,9]$. L'isoniazide est d'abord acétylé en acétylisoniazide qui est très rapidement hydrolysé en acétylhydrazine (et acide isonicotinique) (figure I). L'acétylhydrazine peut être acétylée à son tour en diacétylhydrazine ou être oxydée par le cytochrome $\mathrm{P}-450$ en un métabolite réactif hépatotoxique (figure I). On voit que le phénotype d'acétylation intervient à la fois dans la formation de l'acétylhydrazine et dans son élimination. On comprend que, dans ces conditions, la concentration plasmatique maximale d'acétylhydrazine soit finalement égale chez les acétyleurs lents et rapides. Cependant, chez les acétyleurs lents, la concentration plasmatique d'hydralazine diminue plus lentement. Par conséquent, la quantité de métabolites réactifs formés à partir de l'acétylhydrazine est probablement un peu plus élevée chez les acétyleurs lents. Ceci pourrait expliquer le fait que la prévalence des hépatites dues à l'isoniazide tend à être un peu plus élevée chez ceux-ci [9].

\section{Glucuronoconjugaison}

La glucuronoconjugaison est une des principales biotransformations intéressant à la fois de nombreux médicaments et de nombreuses molécules endogènes telles que la bilirubine et les hormones sexuelles. Les maladies de Gilbert et de Crigler-Najjar sont dues à un déficit héréditaire de la glucuronoconjugaison de la bilirubine [12]. La maladie de Gilbert est une affection fréquente touchant environ I à $2 \%$ des individus. Cette maladie est généralement latente; cependant, elle se manifeste parfois par un ictère dû à une augmentation modérée de la bilirubine non conjuguée, dont la survenue est généralement provqu uée par une infection, le jeûne ou la prise de certains médicaments [12]. Dans la maladie de Gilbert, la diminution de la glucuronoconjugaison est due à un déficit particl d'un enzyme microsomal hépatique, la bilirubine uridine diphosphate glucuronosyltransférase (bilirubine-UDP glucuronosyltransférase) [12, I3]. Ce déficit est probablement transmis comme un caractère autosomique 
dominant [12, 14].

La maladie de Crigler-Najjar est au contraire très rare et se manifeste par un ictère intense apparaissant dans la période néonatale. Cette maladie a été subdivisée en deux types. Dans le type I, le déficit de l'activité bilirubine - UDP glucuronosyltransférase est apparemment complet, et est transmis sur le mode autosomique récessif. Il est improbable que la maladie de Crigler-Najjar de type I soit une forme homozygote de la maladie de Gilbert. Dans le type II, le déficit de l'activité bilirubine - UDP - glucuronosyltransférase est important mais non complet, et est transmis sur le mode autosomique dominant. L'ictère est moins intense que dans le type I [12-I4]. Actuellement, il n'existe pas d'argument prouvant clairement que les maladies de Gilbert et de Crigler-Najjar de type II soient des expressions différentes d'une même maladie. Dans ces maladies héréditaires, le déficit de glucuronoconjugaison affecte principalement la conjugaison de la bilirubine. Cependant la glucuronoconjugaison de certains médicaments est également perturbée (tableau II). Outre le déficit en diverses activités de glucuronoconjugaison, il pourrait exister chez les sujets atteints de maladie de Gilbert d'autres anomalies métaboliques comme une acétylation lente de la sulfadimidine [7], ou une diminution de l'élimination plasmatique de la tolbutamide et de la bromosulfonephtaléine, peut-être par diminution de la captation hépatique [ 15 ].

Le mécanisme du déficit de l'activité de la bilirubine-UDP-glucuronosyltransférase n'est toujours pas établi. Chez l'animal et chez l'homme, on pense qu'il existe plusieurs formes différentes d'UDP glucuronosyltransférase $[7,14]$. Le déficit de la glucuronoconjugaison de la bilirubine et de certains médicaments dans les maladies de Gilbert et de Crigler-Najjar pourrait être dû soit à une anomalie quantitative ou fonctionnelle d'une forme particulière d'UDP - glucuronosyltransférase, soit à une anomalie structurale du support microsomal contenant les enzymes, comme cela a pu être proposé pour la maladie de Crigler-Najjar [7]

\section{Glucoconjugaison}

Récemment mise en évidence, la glucoconjugaison est une voie métabolique essentielle pour certains médicaments comme l'amobarbital [16]. La glucoconjugaison est effectuée par les microsomes hépatiques, en présence d'UDP-glucose. $\mathrm{La}$ formation in vivo du $\mathrm{I}-(\beta-\mathrm{D}-\mathrm{gluco}-$ pyranosyl) amobarbital varie considérablement entre des Canadiens de

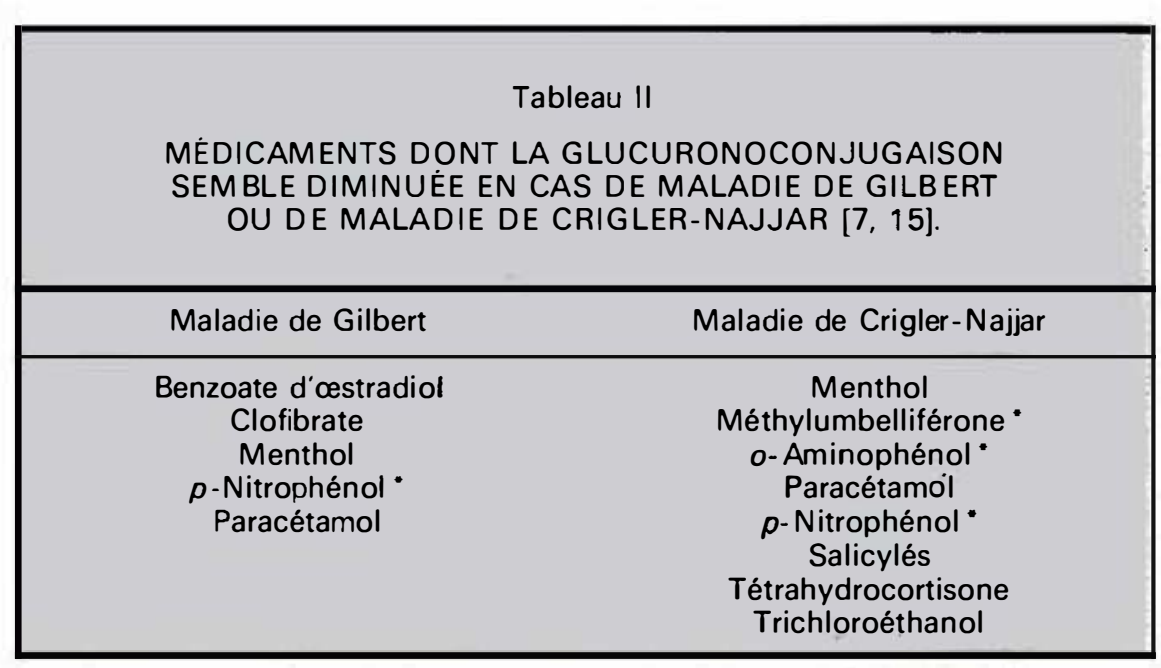

- Médicaments dont la glucuronoconjugaison n'a été étudiée qu'in vitro. $m / s n^{\circ} 7$, vol. 2, septembre 86 différentes ethnies [16] et semble même totalement absente chez de rares sujets $[10,16]$. Ces variations métaboliques suggèrent l'existence d'un polymorphisme génétique.

\section{Les isoenzymes du cytochrome P-450}

L'oxydation de la plupart des médicaments est effectuée par un système microsomal multienzymatique dont l'oxydase terminale est le cytochrome $\mathrm{P}-450$. Cette hémoprotéine est contenue dans de nombreux organes, principalement dans le foie. Il est maintenant bien démontré, tant chez l'homme [I7] que chez l'animal [i8], que le cytochrome $\mathrm{P}-450$ est composé de multiples isoenzymes différents. Ces isoenzymes ont tous un même centre actif, le fer de l'hème sur lequel se fixe l'oxygène qui va oxyder les substrats. Ces isoenzymes diffèrent cependant par la structure de leurs apoprotéines. Ils ont donc des propriétés immunologiques différentes $[17,18]$. Ils ont aussi des propriétés catalytiques différentes. En effet, ces apoprotéines différentes vont ménager, avec les lipides avoisinants du réticulum endoplasmique, des poches hydrophobes différentes ayant des affinités différentes pour divers substrats liposolubles. Un isoenzyme A aura, par exemple, une forte affinité pour un médicament $X$, mais une faible affinité pour un autre médicament Y (figure 2). A l'inverse, un autre isoenzyme $\mathrm{B}$ aura, par exemple, une faible affinité pour le médicament $\mathrm{X}$ mais au contraire une forte affinité pour $\mathrm{Y}$ ( figure 2). Outre ces différences d'affinité, des isoenzymes différents vont pouvoir orienter différemment le substrat en regard du fer de l'hème (figure 2 ). Ainsi, deux isoenzymes différents $A$ et $B$ pourront oxyder un substrat $Z$ en deux positions opposées $(f i$ gure 2).

La synthèse des différents isoenzymes du cytochrome $\mathrm{P}-450$ est régulée séparément. $\mathrm{Un}$ inducteur donné augmente non pas tous les isoenzymes $\mathrm{du}$ cytochrome $\mathrm{P}-450$ mais seulement quelques-uns d'entre eux [18]. Une même sélectivité d'induction est probable chez 


\section{Affinité différente pour divers substrats}
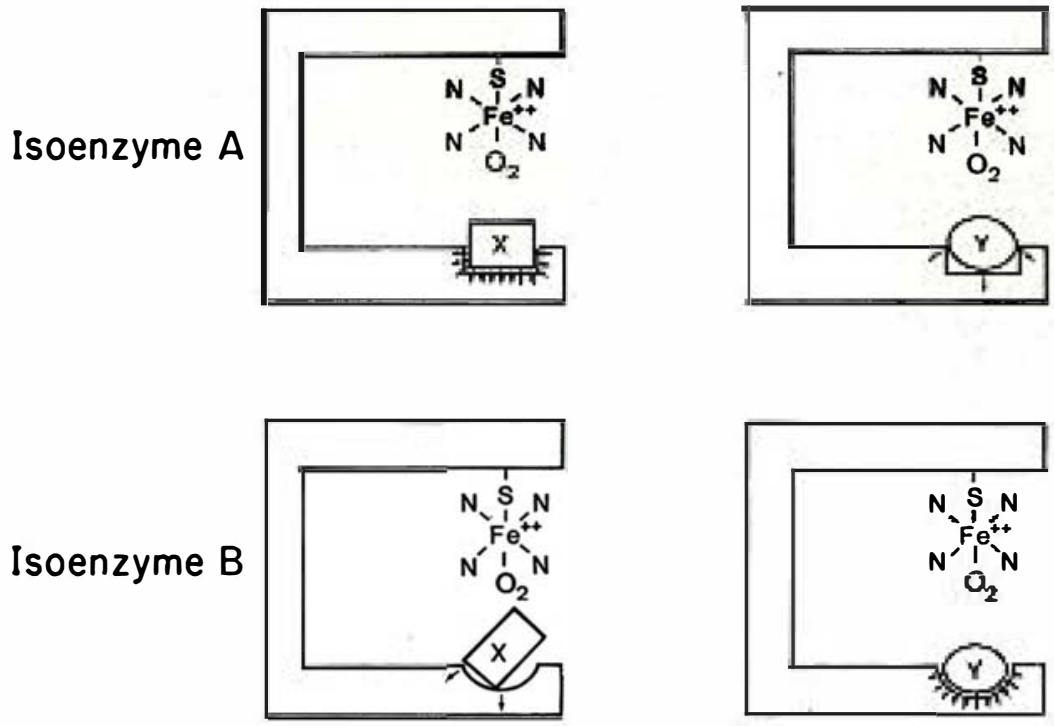

\section{Oxydation en des sites différents}

\section{REFERENCES}

18. Guengerich FP, Dannan GA, Wright T, Martin MV, Kaminsky LS. Purification and characterization of liver microsomal cytochromes $\mathrm{P}-450$ : electrophoretic, spectral, catalytic and immunochemical properties, and inducibility of eight isozymes isolated from rats treated with phenobarbital or $\beta$-napthoflavonc. Biochemistry 1982; 2 r: 6019-30.

19. Otton SV, Inaba T, Kalow W. Inhibition of sparteine oxidation in human liver by tricyclic antidepressants and other drugs. Life Sci 1985 ; 32: 795-80o.

20. Otton SV, Inaba T, Kalow W. Competitive inhibition of spartcine oxidation in human liver by $\beta$-adrenoceptor antagonists and other cardiovascular drugs. Life Sci 1985; 34: 73-80.

21. Birgersson C, Blanck A, Woodhouse $K$, Mellström B, Von Bahr C. Comparative metabolism of debrisoquine, 7-ethoxyresorufin and benzo(a)pyrene in liver microsomes from humans, and from rats treated with cytochromes $\mathrm{P}-450$ inducers. Acta Pharmacol Toxicol (Copenh) 1985; 57: 1 17-20.

22. Roy SD, Hawes EM, McKay G, Korchinski ED, Midha KK. Metabolism of methoxyphenamine in extensive and poor metabolizers of debrisoquine. Clin Pharmacol Ther 1985; 28: 128-33.
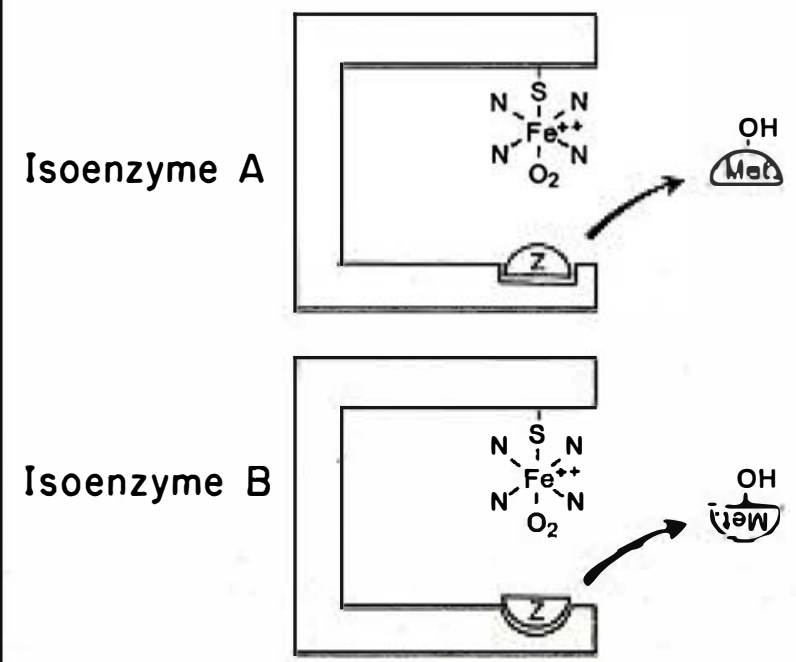

Figure 2. Conséquences de l'existence de divers isoenzymes différents du cytochrome P-450. Le cytochrome P-450 est formé par une molécule d'hème (schématisée par un atome de fer et les 4 azotes du noyau tétrapyrrole) attachée sur un cystéinate d'une apoprotéine (schématisée par la partie rectangulaire). L'oxygène se fixe sur le fer de l'hème. Les substrats se fixent sur une poche hydrophobe ménagée par l'apoprotéine. L'apoprotéine diffère d'un isoenzyme à l'autre. Ces apoprotéines différentes ménagent des poches hydrophobes différentes et ont. de ce fait, des affinités différentes pour des substrats différents. En outre, des isoenzymes différents vont orienter différemment les substrats par rapport au fer de l'hème (donc à l'oxygène), et vont ainsi oxyder les médicaments en des sites différents. 
l'homme : divers inducteurs, tels le phénobarbital, la rifampicine, la troléandomycine ou l'érythromycine, modifient de façon très différente le métabolisme hépatique des médicaments [2]. Le plus souvent, un médicament est transformé en plusieurs métabolites différents (figure 3). Qui plus est, chacun de ces métabolites est lui-même formé par plusieurs isoenzymes du cytochrome $\mathrm{P}-45^{\circ}$ (figure 3); notons cependant qu'un même isoenzyme peut participer à la formation de plusieurs métabolites différents (figure 3). Pour un tel médicament, le déficit isolé d'un isoenzyme donné peut diminuer la formation d'un des métabolites mais n'aura guère d'effet sur la clairance globale du médicament. Celle-ci est en effet déterminée par l'activité de multiples isoenzymes et donc, initialement, par de multiples gènes différents. Les variations de ce système polygénique aboutissent en général à une distribution régulière, unimodale, des clairances ( figure 3). C'est ainsi que la distribution de la clairance de l'antipyrine est en première approximation unimodale. $\mathrm{Si}$ l'on effectue cependant une analyse très fine de cette distribution, on peut arriver à faire apparaître un polymorphisme discret qui devient plus net lorsque l'on étudie séparément la variation de chaque métabolite [7]. Pour d'autres médicaments, un seul métabolite principal est formé d'une part et, d'autre part, ce métabolite principal n'est formé que par un seul isoenzyme du cytochrome $\mathrm{P}-450$ ( figure 3). Dans ce cas particulier, toute variation quantitative ou qualitative de cet isoenzyme va modifier significativement le métabolisme et donc la clairance du médicament; si l'isoenzyme est déficient chez certains sujets, la clairance aura alors une distribution polymodale, avec des métaboliseurs actifs, faibles, et parfois intermédiaires (figure 3 ).

\section{Hydroxylation de la débrisoquine}

La débrisoquine est un médicament sympatholytique, utilisé en Grande-Bretagne dans le traitement de l'hypertension artérielle. La débri-

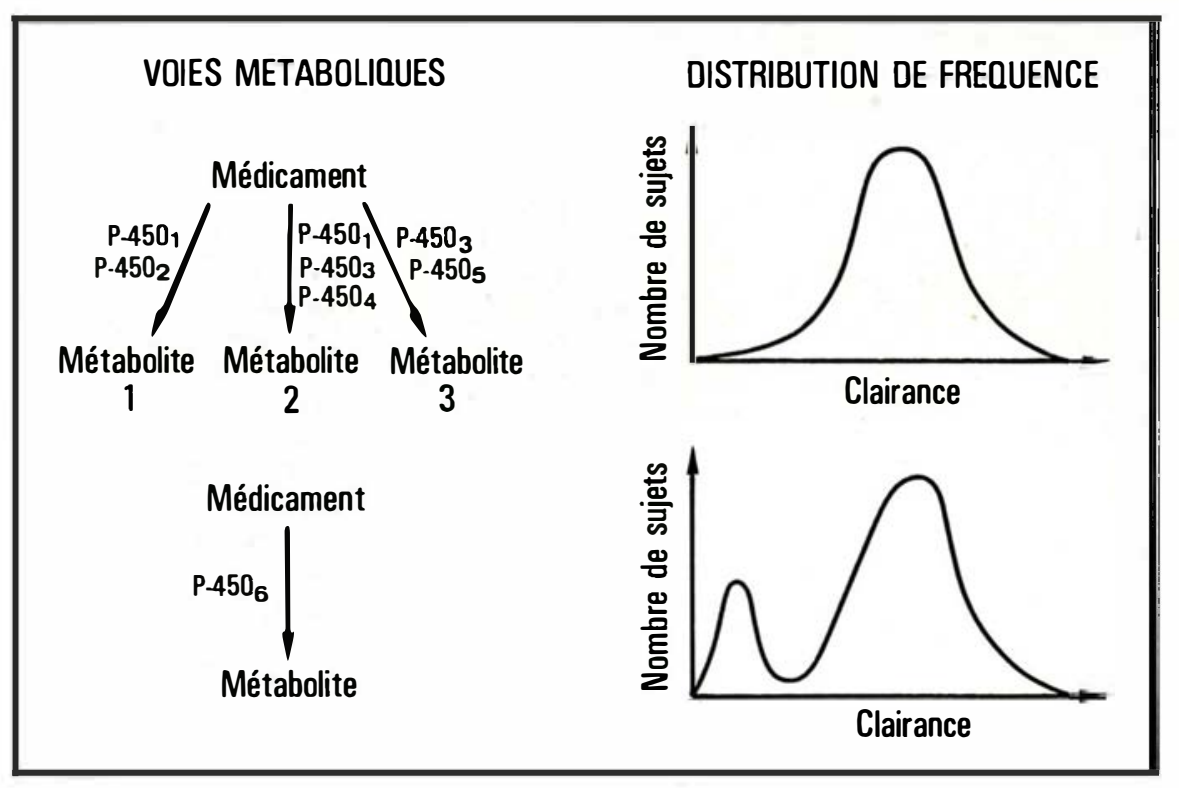

Figure 3. Relation entre voies métaboliques et distribution de fréquence. Certains médicaments sont oxydés en plusieurs métabolites formés chacun par plusieurs isoenzymes différents du cytochrome P-450; l'intervention de multiples isoenzymes aboutit à une distribution polygénique, unimodale de la clairance globale du médicament. Pour quelques médicaments, un seul métabolite principal est formé par un seul isoenzyme; le déficit de cet isoenzyme chez certains sujets aboutit à un polymorphisme génétique avec une distribution bimodale (ou parfois trimodale) de la clairance.

soquine est principalement métabolisée par oxydation en 4-hydroxydébrisoquine. Ce métabolite, ainsi que la débrisoquine non transformée, sont éliminés dans les urines. En I977, Maghoub et coll. [4] ont montré que l'efficacité de la 4-hydroxylation de la débrisoquine a une distribution bimodale dans la population anglaise. Alors que la majorité des sujets hydroxylent fortement la débrisoquine et ont de ce fait été appelés métaboliseurs actifs, un petit nombre de sujets forment, au contraire, très peu de 4-hydroxydébrisoquine et par conséquent éliminent lentement la débrisoquine; ces sujets ont été appelés métaboliseurs faibles [4]. Les études de transmission génétique ont montré que la 4-hydroxylation de la débrisoquine est contrôlée par une paire d'allèles; le caractère métaboliseur faible est autosomique récessif [5-7]. La fréquence du phénotype métaboliseur faible diffère beaucoup d'une population à l'autre. Elle est basse chez les Égyptiens et les Arabes Saoudiens ( $1 \%$ ), comprise entre 3 et Io $\%$ chez les Américains du Nord et chez les Européens et elle atteint $30 \%$ chez les Chinois de Hongkong [5, 6, Io]. Il est intéressant d'observer que dans certaines populations, il semble exister un phénotype intermédiaire pouvant correspondre aux hétérozygotes [7]. Parallèlement aux travaux anglais sur la débrisoquine, Eichelbaum et coll. [5] ont montré l'existence d'un déficit de la $\mathrm{N}$-oxydation de la spartéine, un médicament ocytocique et anti-arythmique; ce déficit était observé chez cinq pour cent des Allemands [5]. Rapidement, il est apparu que le déficit d'oxydation de la spartéine était associé au déficit d'hydroxylation de la débrisoquine et inversement, suggérant qu'il s'agissait probablement du même polymorphisme [5-7]. Le polymorphisme de l'hydroxylation de la débrisoquine et de la spartéine affecte également le métabolisme 

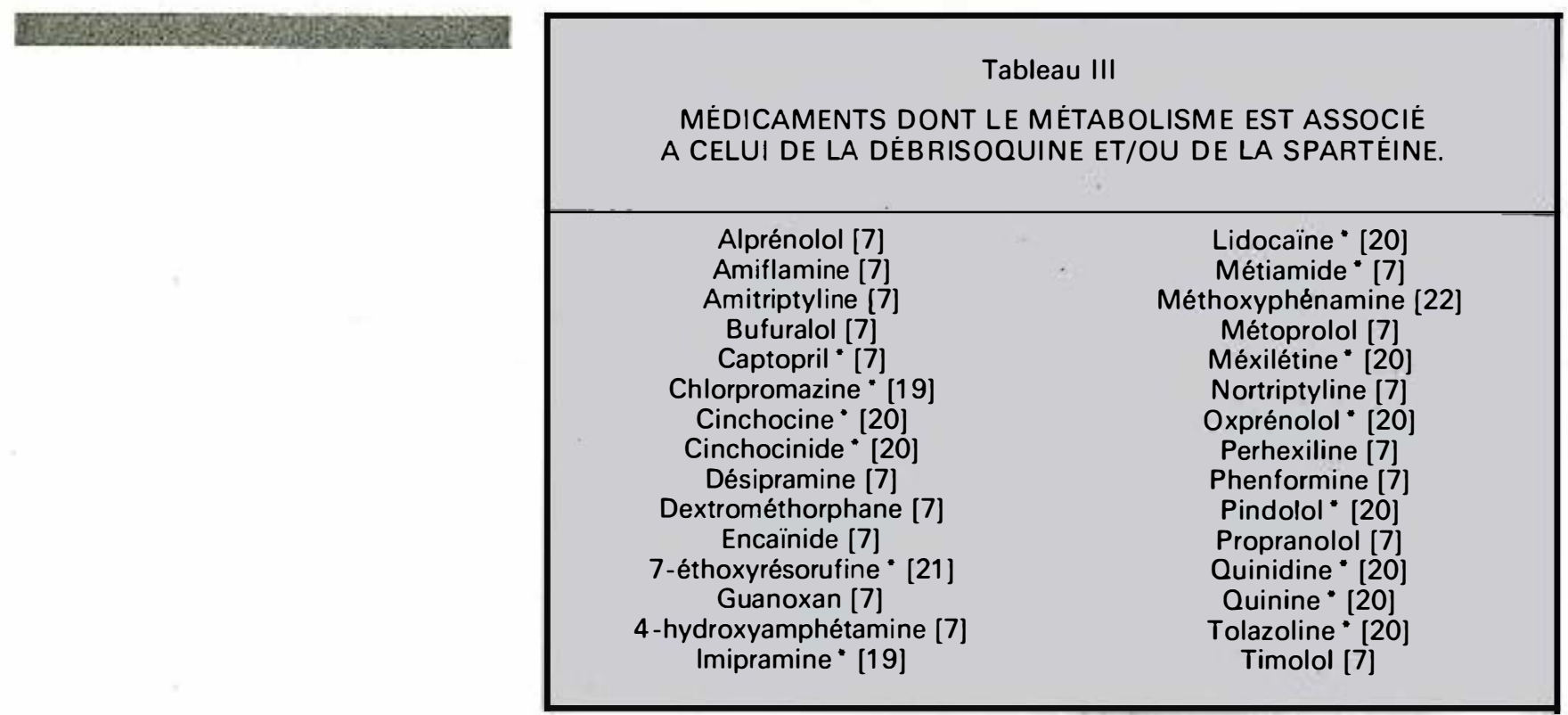

- Association possible mais non définitivement démontrée avec le métabolisme de la débrisoquine et de la spartéine.

\section{REFERENCES}

23. Larrey $D$, Distlerath LM, Dannan GA Wilkinson GR, Guengerich FP. Purification and characterization of the rat liver microsomal cytochrome $\mathrm{P}-450$ involved in the 4-hydroxylation of debrisoquine, a prototype for genetic variation in oxidative drug membolism. Biochemistry 1984; 23: 2787-95.

24. Distlerath LM, Reilly PEB, Martin MV, Davis GG, Wilkinson GR, Guengerich FP. Purification and characterization of the human liver cytochromes $\mathrm{P}-45^{\circ}$ involved in debrisoquine 4-hydroxylation and phenacetin O-deethylation. Two prototypes for genetic polymorphism in oxidative drug metabolism. $7 \mathrm{Biol}$ Chem $1985 ; 260$ : 9057-67.

25. Vasco MR, Bell RD, Daly DD, Pippenger $\mathrm{CE}$. Inheritance of phenytoin hypometabolism: a kinetic study of one family. Clin Pharmacol Ther 1980; 27: 96-103.

26. Kahn GC, Boobis AR, Brodie MJ, Toverud E-L, Murray S, Davics DS. Phenacetine O-deethylase: an activity of a cytochrome P-450 showing genetic linkage with that catalysing the 4-hydroxylation of debrisoquinc? $\mathrm{Br} \mathrm{J}$ Clin Pharmacol 1985; 20: 67-76.

27. Shimada T, Guengerich FP. Participation of a rat liver cytochrome $\mathrm{P}-450$ induced by pregnenolone $16 \alpha$-carbonitrile and other compounds in the 4-hydroxylation of mephenytoin. Mol Pharmacol d'une trentaine d'autres médicaments (tableau III). En revanche, le métabolisme de nombreux autres médicaments n'est pas lié à celui de la débrisoquine. C'est le cas, par exemple, de l'antipyrine [5-7].

\section{Détermination du phénotype}

Le phénotype de l'oxydation de la débrisoquine est le plus souvent déterminé par le calcul du "quotient métabolique ". Celui-ci a été arbitrairement défini comme étant égal au rapport débrisoquine/4 - hydroxydébrisoquine présentes dans les urines recueillies pendant les huit heures suivant l'ingestion de Io $\mathrm{mg}$ de débrisoquine [4]. Notons que le quotient dit "métabolique" ainsi défini est élevé lorsque le métabolisme est faible : le phénotype métaboliseur faible est caractérisé par un quotient métabolique supérieur à I 2,6 [5-7]. Cette méthode a certaines limites. En effet, certains médicaments comme le guanoxan, la spartéine et la phenformine [7] peuvent inhiber la 4 -hydroxylation de la débrisoquine. En outre, la carbamazépine semble également majorer le quotient métabolique [7].
Par conséquent, la prise de ces medicaments et plus généralement de ceux dont le métabolisme est associé à celui de la débrisoquine doit être interrompue lors du test afin d'éviter de qualifier de métaboliseur faible un métaboliseur actif dont le métabolisme est inhibé. Le phénotype peut également être déterminé avec la spartéine suivant le même principe [5]. Cependant, l'absence de méthode simple de dosage de ces deux médicaments a fait proposer récemment de nouvelles méthodes utilisant d'autres médicaments comme le métoprolol, le bufuralol ou le dextrométhorphane [5-7].

\section{Mécanisme \\ biochimique}

Il a été montré que l'activité débrisoquine 4-hydroxylase est une activité monooxygénasique du cytochrome $\mathrm{P}-450$ hépatique. Cette activité est fortement diminuée dans les microsomes hépatiques de métaboliseurs faibles, alors que le cytochrome $\mathrm{P}-450$ total et plusieurs autres activités monooxygénasiques ne sont pas diminués. Cela suggère fortement une anomalie d'un isoenzyme particulier ne représentant 
qu'une petite fraction de l'ensemble du cytochrome $\mathrm{P}-450$ mais cependant responsable presque exclusivement de l'oxydation de la débrisoquine [5-7].

Un modèle de polymorphisme génétique du métabolisme de la débrisoquine a été récemment identifié chez le rat. Les femelles de rat DA métabolisent très faiblement la débrisoquine en 4-hydroxydébrisoquine [23]. Il vient d'être démontré que ce déficit métabolique est dû au déficit quantitatif d'un isoenzyme du cytochrome $\mathrm{P}-450$ hépatique, le cytochrome $\mathrm{P}-450 \mathrm{UT}-\mathrm{H}$, qui est responsable de $90 \%$ de la 4-hydroxylation de la débrisoquine [23]. Il est intéressant de constater que l'anticorps anti-P-450 UT-H inhibe également l'isoenzyme responsable de l'oxydation de la débrisoquine chez l'homme [24]. L'isoenzyme du cytochrome P-450 humain (cytochrome $\mathrm{P}-450 \mathrm{Db}$ ) responsable de l'activité débrisoquine 4-hydroxylase vient d'être identifié [24]. Cependant, le mécanisme par lequel son activité est diminuée est encore hypothétique.

\section{Conséquences cliniques}

L'existence d'un polymorphisme génétique dans le métabolisme d'un médicament n'a pas nécessairement de conséquence clinique. Un exemple en est le propranolol dont la 4-hydroxylation est déficiente chez les métaboliseurs faibles [7]. Cependant, cette voie métabolique étant mineure, sa déficience n'entraine pas de diminution de la clairance globale du propranolol. De ce fait, pour une dose standard de propranolol, le blocage $\beta$-adrénergique n'est pas augmenté chez les métaboliseurs faibles [7]. Un autre exemple est celui de l'amitriptyline. Bien que la Io-hydroxylation de l'amitriptyline soit déficiente chez les métaboliseurs faibles, la clairance de ce médicament n'est pas diminuée, du fait de son élimination prédominante par $\mathbf{N}$-déméthylation [7].

Dans de nombreux autres cas cependant, le déficit d'hydroxylation a des conséquences cliniques néfastes. Selon que c'est le médicament lui-même ou l'un de ces méta- bolites qui est actif ou toxique, diverses conséquences sont prévisibles. Une première conséquence est la possibilité d'un effet thérapeutique exagéré. C'est ainsi que chez les métaboliseurs faibles, la débrisoquine entraîne des hypotensions sévères; le bufuralol, le timolol et l'alprenolol entraînent un blocage $\beta$-adrénergique excessif; la phenformine peut entraîner une acidose lactique [5-7]. Un deuxième aspect est une toxicité accrue du médicament non transformé. L'exemple est ici la perhexiline qui s'accumule excessivement chez les métaboliseurs faibles et peut ainsi entraîner une phospholipidose lysosomale, des hépatites ou des neuropathies périphériques $[6,7]$. Une troisième possibilité est celle d'une diminution de l'effet thérapeutique chez les métaboliseurs faibles. L'exemple en est l'encaïnide, dont les propriétés antiarythmiques sont dues à l'un de ses métabolites, qui est l'Odesméthylencaïnide. Chez les métaboliseurs faibles, la production de ce métabolite est très diminuée. Il en résulte une forte diminution de l'effet thérapeutique [7].

Finalement, il convient de remarquer que le fait d'être un métaboliseur faible n'est pas toujours néfaste. C'est ainsi que le risque d'agranulocytose au métiamide pourrait être moindre chez les métaboliseurs faibles, probablement du fait d'une moindre formation d'un métabolite toxique [5-7]. Il a même été rapporté que le risque de cancer digestif ou de cancer bronchique pourrait être plus faible chez les métaboliseurs faibles de la débrisoquine, peut-être du fait d'une moindre formation de métabolites cancérigènes [7]. Ces observations nécessitent confirmation.

\section{Autres polymorphismes d'oxydation}

Le métabolisme de la phénytoïne [25], de la tolbutamide, de la méphénytoïne, de la carbocystéine, de la nifédipine [7] et de la phénacétine [26] est également l'objet d'un polymorphisme génétique. Ces polymorphismes sont différents de celui de la débrisoquine. On ne sait encore s'ils sont tous différents en- tre eux. Il faut noter que le déficit de l'oxydation de la phénacétine a pour conséquence une toxicité accrue d'un métabolite formé par une autre voie. En effet, chez les métaboliseurs faibles de la phénacétine, il existe une diminution de la $\mathrm{N}$-dééthylation oxydative de la phénacétine en paracétamol. Il en résulte une production accrue d'un autre métabolite, la 2-hydroxyphénétidine, qui peut entraîner une méthémoglobinémie [7]. Les isoenzymes du cytochrome $\mathrm{P}-450$ hépatique responsables de la 4-hydroxylation de la méphénytoine chez le rat [27] et de la O-dééthylation de la phénacétine chez l'homme [24], viennent d'être isolés. Le mécanisme responsable du déficit de l'activité de ces isoenzymes est encore inconnu.

\section{Conclusions et perspectives}

Certains isoenzymes du cytochrome P-450 catalysent très sélectivement l'oxydation d'un substrat endogène donné, comme le cholestérol ou un stéroïde. Un déficit d'un tel isoenzyme est soit incompatible avec la vie, soit marqué par une maladie. A l'inverse, d'autres isoenzymes semblent essentiellement là pour métaboliser les xénobiotiques. Les variations de concentration ou même de structure de tels isoenzymes passent inaperçues tant que le sujet n'absorbe pas certains médicaments; les mutations n'étant pas sanctionnées, la voie est ouverte à de multiples variations génétiques.

Jusqu'à ces dernières années, on connaissait surtout les variations polygéniques, unimodales. Dans un tel système, il est impossible de prévoir le métabolisme d'un médicament donné avant de l'avoir étudié. $\mathrm{La}$ survenue de réactions dites "idiosyncrasiques" était considérée comme une fatalité inévitable. La découverte accélérée, au cours des dernières années, de divers polymorphismes affectant chacun de multiples médicaments (tableaux $I$ à III) amène à réviser, en partie, cette façon de penser. Il devient en effet possible, en déterminant quelques phénotypes, de prévoir le métabolisme d'un 
grand nombre de médicaments. Actuellement, les doses usuelles d'un médicament sont déterminées à partir de la population globale. Elles sont donc adaptées au phénotype le plus fréquent. Elles sont, par contre, monstrueusement inadaptées au phénotype rare, par exemple au phénotype "métaboliseur faible " de la débrisoquine. Ces sujets sont exposés au risque de surdosage ou d'autres complications iatrogènes. Bien que minoritaires, ils représentent cependant de l'ordre de 5 millions de Français. A l'évidence, nous devons prendre les mesures nécessaires pour les protéger.

(a) Des efforts importants méritent d'être consacrés à la recherche d'un polymorphisme génétique à l'origine des accidents iatrogènes les plus fréquents. Notons que ces recherches sont indiquées quelle que soit la nature, toxique ou allergique, des complications. Comme le montre en effet l'exemple du lupus érythémateux médicamenteux dont l'incidence est accrue chez l'acétyleur lent, il existe bel et bien une relation entre concentration du médicament et survenue de manifestations immunitaires.

(b) Pour les nouveaux médicaments, il semblerait souhaitable que, dans l'avenir, la recherche préalable d'un polymorphisme métabolique, et éventuellement, la détermination des posologies adaptées à chaque phénotype, soient exigées pour l'autorisation de mise sur le marché.

(c) Il est envisageable que, dans la décennie à venir, il devienne aussi fréquent de demander un phénotype d'acétylation, d'hydroxylation ou autre que de déterminer un groupe sanguin. Des recherches méritent d'être poursuivies pour déterminer les substrats les plus pratiques pour une telle détermination.

(d) Dès à présent, il semblerait logique de déterminer le phénotype d'hydroxylation avant l'administration de certains médicaments dangereux, comme la perhexiline. La possibilité de prévenir certains accidents iatrogènes est un grand espoir pour le médecin, mais lui confère en contrepartie une responsabilité

\begin{abstract}
accrue
\end{abstract}

\section{Summary}

The elimination of many endoand xenobiotics requires their biotransformation into more soluble metabolites, mostly in the liver. The influence of genetic control on drug biotransformation gave birth to a new discipline, pharmacogenetics. Variations in monogenic controls lead to polymodal distributions of the metabolic phenotypes in population, with extensive metabolizers, poor metabolizers, and sometimes intermediate metabolizers. The main examples of genetic polymorphism of hepatic metabolism are $\mathrm{N}$-acetylation, glucuronoconjugation and oxidation mediated by cytochrome $\mathrm{P}_{-}-45^{\circ}$ isozymes. The standard doses of drugs are calculated from the global population, and are adapted to subjects with common metabolic phenotypes. In contrast, they are markedly inadapted to those with uncommon metabolic phenotypes, for instance, the phenotype "poor metabolizer" of debrisoquine. Consequently, the latter subjects are exposed to overdose, and other drug-induced adverse reactions. Prevention of these drug-induced adverse reactions requires: $(a)$ a better understanding of mechanisms responsible for genetic polymorphism at enzyme level; $(b)$ phenotyping patients who should receive drugs with known genetic polymorphism and responsible for severe complications; $(c)$ detecting genetic polymorphism for drugs inducing idiosyncratic reactions.

\section{TIRES A PART
D. Larrey : Unité de recherches de physiopatho-
logie hépatique, Inserm U 24, hôpital Beaujon,
92 1 8 Clichy Cedex. \\ TIRES A PART
D. Larrey : Unité de recherches de physiopatho-
logie hépatique, Inserm U 24, hôpital Beaujon,
92 1 8 Clichy Cedex. \\ TIRES A PART
D. Larrey : Unité de recherches de physiopatho-
logie hépatique, Inserm U 24, hôpital Beaujon,
92 1 8 Clichy Cedex.}

